\title{
Heck-type Arylation of 2-Cycloalken-1-ones with Arylpalladium Intermediates formed by Decarboxylative Palladation and by Aryl Iodide Insertion
}

\author{
Daisuke Tanaka and Andrew G. Myers* \\ Department of Chemistry and Chemical Biology, Harvard University, \\ Cambridge, Massachusetts 02138
}

\section{Experimental Procedures and Spectral Data}

Commercial materials $\left(\mathrm{Pd}\left(\mathrm{O}_{2} \mathrm{CCF}_{3}\right)_{2}, \mathrm{Pd}(\mathrm{OAc})_{2}, \mathrm{Ag}_{2} \mathrm{CO}_{3}\right)$ were purchased and used as received. $\mathrm{Pd}\left(\mathrm{O}_{2} \mathrm{CCF}_{3}\right)_{2}, \mathrm{Pd}(\mathrm{OAc})_{2}$, and $\mathrm{Ag}_{2} \mathrm{CO}_{3}$ were purchased from Aldrich or Strem and were used as received. Analytical thin-layer chromatography was performed using glass plates pre-coated with $0.25 \mathrm{~mm} \mathrm{230-400} \mathrm{mesh} \mathrm{silica} \mathrm{gel} \mathrm{impregnated} \mathrm{with} \mathrm{a}$ fluorescent indicator. TLC plates were visualized by exposure to ultraviolet light, or by treatment with ethanolic ceric ammonium molybdate (CAM) or phosphomolybdic acid (PMA) solution, followed by heating on a hot plate. Organic solutions were concentrated by rotary evaporation at $\sim 25$ torr (house vacuum). Flash column chromatography was performed as described by Still et al. ${ }^{1}$ HPLC analysis was performed on a Beckmann Ultrasphere ODS 4.6\25 mm reverse-phase column with an acetonitrile/methanol/trifluoroacetic acid/water eluent system. Peaks were detected by absorption intensities at 260 and $300 \mathrm{~nm}$. 4-Methoxybiphenyl was employed as an internal standard.

Proton nuclear magnetic resonance spectra $\left({ }^{1} \mathrm{H}\right.$ NMR) and carbon nuclear magnetic resonance spectra $\left({ }^{13} \mathrm{C}\right.$ NMR) were acquired on a Varian Mercury 400 (400 $\mathrm{MHz}$ ) NMR spectrometer. Chemical shifts for protons are reported in parts per million ( $\square$ downfield from tetramethylsilane (TMS), referenced to residual protium in the NMR solvent. Chemical shifts for carbon are reported in parts per million ( $\square$ ) downfield from TMS and are referenced to the carbon resonance of the solvent $\left(\square \mathrm{CDCl}_{3}, 77.0\right)$. IR spectra were obtained using a Perkin-Elmer 1600 FT-IR spectrophotometer. Melting points were obtained with a Thomas Hoover Uni-Melt Capillary Melting Point Apparatus and are uncorrected. Low- and high-resolution mass spectra were obtained at the Harvard University Mass Spectrometry Facilities.

Physical data for 3-(2,4-dimethoxyphenyl)-2-cyclohexen-1-one and 3-(2,4,6trimethylphenyl)-2-cyclohexen-1-one have been previously reported. ${ }^{2}$ 3-(4Methylphenyl)-2-cyclohexen-1-one was identified by comparison to published NMR spectra $\left({ }^{1} \mathrm{H}\right.$ and $\left.{ }^{13} \mathrm{C}\right)$ and MS analysis. ${ }^{3}$ 4-Isopropyl-2-cyclohexen-1-one was prepared by a reported method. ${ }^{4}$

\footnotetext{
${ }^{1}$ Still, W.C.; Kahn, M.; Mitra, A. J. Org. Chem. 1978, 43, 2923.

${ }^{2}$ Myers, A. G., Tanaka, D., Mannion, M. R., J. Am. Chem. Soc., 2002, 124, 11250.

${ }^{3}$ Cho, C. S., Motofusa, S., Ohe, K., Uemura, S., J. Org. Chem., 1995, 60, 883.

${ }^{4}$ Queiroga, C. L., Ferracini, V. L., Marsaioli, A. J., Phytochemistry, 1996, 42, 1097.
} 
An Illustrative Procedure. A mixture of 2,4-dimethoxybenzoic acid (0.182 g, 1.00 mmol, 1.00 equiv), 4-isopropyl-2-cyclohexen-1-one ( $0.216 \mathrm{~g}, 1.56 \mathrm{mmol}, 1.56$ equiv), palladium (II) trifluoroacetate $(0.067 \mathrm{~g}, 0.20 \mathrm{mmol}, 0.20$ equiv), and silver (I) carbonate (0.552 g, $2.00 \mathrm{mmol}, 2.00$ equiv) in 5\% DMSO-DMF $(4.2 \mathrm{~mL})$ was heated at $80{ }^{\circ} \mathrm{C}$ under air. After $90 \mathrm{~min}$, the reaction mixture was allowed to cool, and the cooled solution was diluted with ethyl acetate $(30 \mathrm{~mL})$. The resulting dark suspension was filtered through a Celite pad. The filtrate was washed sequentially with an aqueous solution of hydrochloric acid $(1 \mathrm{M}, 10 \mathrm{~mL})$ and water $(5 \square 10 \mathrm{~mL})$. The product solution was dried over $\mathrm{MgSO}_{4}$, the dried solution was filtered, and the filtrate was concentrated. Purification of the residue by flash column chromatography (1:5 ethyl acetate in hexanes) afforded 4-isopropyl-3-(2,4-dimethoxyphenyl)-2-cyclohexen-1-one $(0.235 \mathrm{~g}, 0.857 \mathrm{mmol}, 86 \%)$ as an oil.

All compounds were prepared analogously with the above procedure.

3-(2,6-Dimethoxyphenyl)-2-cyclohexen-1-one (Table 1, entry 2)

$\mathrm{R}_{f} 0.7$ (hexanes:EtOAc $\left.=1: 1\right) ; \mathrm{mp}=91-92{ }^{\circ} \mathrm{C} ;{ }^{1} \mathrm{H}$ NMR $\left(400 \mathrm{MHz}, \mathrm{CDCl}_{3}\right) \square 7.23(\mathrm{t}$, $1 \mathrm{H}, J=8.4 \mathrm{~Hz}), 6.57(\mathrm{~d}, 2 \mathrm{H}, J=8.4 \mathrm{~Hz}), 5.97(\mathrm{t}, 1 \mathrm{H}, J=1.6 \mathrm{~Hz}), 3.76(\mathrm{~s}, 6 \mathrm{H}), 2.53(\mathrm{dt}$, $2 \mathrm{H}, J=1.5,5.9 \mathrm{~Hz}), 2.48(\mathrm{t}, 2 \mathrm{H}, \mathrm{J}=6.8 \mathrm{~Hz}), 2.14-2.08(\mathrm{~m}, 2 \mathrm{H}) ;{ }^{13} \mathrm{C} \mathrm{NMR}(100 \mathrm{MHz}$, $\left.\mathrm{CDCl}_{3}\right) \square 200.0,158.1,156.6,130.1,129.4,118.6,103.9,55.8,37.5,30.2$, 23.0; FTIR $\left(\mathrm{CHCl}_{3}\right) \mathrm{cm}^{-1} 3010,1661,1588,1472,1434,1252,1112$; HRMS (EI) $\mathrm{m} / z$ calcd for $\mathrm{C}_{14} \mathrm{H}_{16} \mathrm{O}_{3}$ 232.1100, found 232.1094.

3-(2,6-Difluorophenyl)-2-cyclohexen-1-one (Table 1, entry 4)

$\mathrm{R}_{f} 0.6$ (hexanes:EtOAc $\left.=2: 1\right) ;{ }^{1} \mathrm{H}$ NMR $\left(400 \mathrm{MHz}, \mathrm{CDCl}_{3}\right) \square 7.31-7.24(\mathrm{~m}, 1 \mathrm{H})$, 6.94-6.89 (m, 2H), $6.14(\mathrm{~s}, 1 \mathrm{H}), 2.64(\mathrm{t}, 2 \mathrm{H}, J=5.9 \mathrm{~Hz}), 2.50(\mathrm{t}, 2 \mathrm{H}, J=6.6 \mathrm{~Hz})$, 2.17-2.11 (m, 2H); ${ }^{13} \mathrm{C}$ NMR (100 MHz, $\left.\mathrm{CDCl}_{3}\right) \square 199.0,159.3(\mathrm{dd}, J=7.3,250 \mathrm{~Hz}$ ), 150.9, 131.5, $130.2(\mathrm{t}, J=10.6 \mathrm{~Hz}), 111.8(\mathrm{~d}, J=13.2 \mathrm{~Hz}), 111.8(\mathrm{~d}, J=25.6 \mathrm{~Hz}), 37.3$, 29.9 (t, $J=2.6 \mathrm{~Hz}$ ), 22.9; FTIR (neat film) $\mathrm{cm}^{-1}$ 2944, 1673, 1626, 1464, 1346, 1231, 1001, 786, 722; HRMS (EI) $\mathrm{m} / \mathrm{z}$ calcd for $\mathrm{C}_{12} \mathrm{H}_{10} \mathrm{~F}_{2} \mathrm{O} 208.0700$, found 208.0692.

3-(3-Bromo-2,6-dimethoxyphenyl)-2-cyclohexen-1-one (Table 1, entry 5)

$\mathrm{R}_{f} 0.5$ (hexanes:EtOAc = 2:1); ${ }^{1} \mathrm{H}$ NMR $\left(400 \mathrm{MHz}, \mathrm{CDCl}_{3}\right) \square 7.42(\mathrm{~d}, 1 \mathrm{H}, J=9.2 \mathrm{~Hz})$, $6.59(\mathrm{~d}, 1 \mathrm{H}, J=8.8 \mathrm{~Hz}), 5.98(\mathrm{t}, 1 \mathrm{H}, J=1.5 \mathrm{~Hz}), 3.74(\mathrm{~s}, 3 \mathrm{H}), 3.71(\mathrm{~s}, 3 \mathrm{H}), 2.55-2.52$ $(\mathrm{m}, 2 \mathrm{H}), 2.48(\mathrm{t}, 2 \mathrm{H}, J=6.8 \mathrm{~Hz}), 2.14-2.07(\mathrm{~m}, 2 \mathrm{H}) ;{ }^{13} \mathrm{C} \mathrm{NMR}\left(100 \mathrm{MHz}, \mathrm{CDCl}_{3}\right) \square$ 199.4, 157.0, 156.0, 153.9, 132.8, 130.1, 125.6, 108.3, 108.2, 61.6, 56.0, 37.4, 30.4, 23.1; FTIR (neat film) $\mathrm{cm}^{-1} 2939,1668,1463,1403,1283,1233,1090,800$; HRMS (TOF) $\mathrm{m} / \mathrm{z}$ calcd for $\mathrm{C}_{14} \mathrm{H}_{15} \mathrm{BrO}_{3} 311.0283$, found 311.0278 .

3-(4,5-Dimethoxy-2-nitrophenyl)-2-cyclohexen-1-one (Table 1, entry 6)

$\mathrm{R}_{f} 0.2$ (hexanes:EtOAc = 1:1); ${ }^{1} \mathrm{H}$ NMR $\left(400 \mathrm{MHz} \mathrm{CDCl}_{3}\right) \square 7.65(\mathrm{~s}, 1 \mathrm{H}), 6.62(\mathrm{~s}, 1 \mathrm{H})$, $5.92(\mathrm{~s}, 1 \mathrm{H}), 3.93(\mathrm{~s}, 6 \mathrm{H}), 2.49-2.44(\mathrm{~m}, 4 \mathrm{H}), 2.20-2.14(\mathrm{~m}, 2 \mathrm{H}) ;{ }^{13} \mathrm{C}$ NMR $(100 \mathrm{MHz}$, $\left.\mathrm{CDCl}_{3}\right) \square 199.0,161.9,153.4,148.7,138.5,131.2,127.0,110.9,107.8,56.5,56.4,37.2$, 30.9, 23.2; FTIR (neat film) $\mathrm{cm}^{-1} 2944,1668,1574,1520,1336,1279,1223,1086$, 
1008, 797; HRMS (TOF) $m / z$ calcd for $\mathrm{C}_{14} \mathrm{H}_{15} \mathrm{NO}_{5} 278.1028$, found 278.1024.

3-(2,4-Dimethoxypyridin-3-yl)-2-cyclohexen-1-one (Table 1, entry 7)

$\mathrm{R}_{f} 0.8$ (hexanes:EtOAc $\left.=2: 1\right) ; \mathrm{mp}=78-79{ }^{\circ} \mathrm{C} ;{ }^{1} \mathrm{H}$ NMR $\left(400 \mathrm{MHz}, \mathrm{CDCl}_{3}\right) \square 7.51(\mathrm{~d}$, $1 \mathrm{H}, J=8.1 \mathrm{~Hz}), 6.38(\mathrm{~s}, 1 \mathrm{H}), 6.33(\mathrm{~d}, 1 \mathrm{H}, J=7.8 \mathrm{~Hz}), 3.96(\mathrm{~s}, 3 \mathrm{H}), 3.93(\mathrm{~s}, 3 \mathrm{H}), 2.71$ $(\mathrm{t}, 2 \mathrm{H}, J=6.0 \mathrm{~Hz}), 2.44(\mathrm{t}, 2 \mathrm{~h}, J=6.6 \mathrm{~Hz}), 2.11-2.04(\mathrm{~m}, 2 \mathrm{H}) ;{ }^{13} \mathrm{C}$ NMR $(100 \mathrm{MHz}$, $\left.\mathrm{CDCl}_{3}\right) \square 200.1,163.3,159.9,158.1,140.0,127.0,113.8,101.4,53.7,53.5,37.3,29.3$, 23.1; FTIR $\left(\mathrm{CHCl}_{3}\right) \mathrm{cm}^{-1} 3011,2948,1658,1593,1482,1386,1320,1248,1016$; HRMS (TOF) $m / z$ calcd for $\mathrm{C}_{13} \mathrm{H}_{15} \mathrm{NO}_{3} 234.1130$, found 234.1122 .

3-(3-Methylbenzofuran-2-yl)-2-cyclohexen-1-one (Table 1, entry 8)

$\mathrm{R}_{f} 0.5$ (hexanes:EtOAc $\left.=2: 1\right) ; \mathrm{mp}=136{ }^{\circ} \mathrm{C} ;{ }^{1} \mathrm{H}$ NMR $\left(400 \mathrm{MHz}, \mathrm{CDCl}_{3}\right) \square 7.56-7.53$ $(\mathrm{m}, 1 \mathrm{H}), 7.44(\mathrm{~d}, 1 \mathrm{H}, J=8.1 \mathrm{~Hz}), 7.35(\mathrm{ddd}, 1 \mathrm{H}, 1.1,7.3,7.3 \mathrm{~Hz}), 7.26(\mathrm{ddd}, 1 \mathrm{H}, J=$ 1.1, 7.4, 7.4 Hz), $6.55(\mathrm{~s}, 1 \mathrm{H}), 2.91-2.87(\mathrm{~m}, 2 \mathrm{H}), 2.50(\mathrm{t}, 2 \mathrm{H}, J=6.8 \mathrm{~Hz}), 2.46(\mathrm{~s}, 3 \mathrm{H})$, 2.19-2.13 (m, 2H); ${ }^{13} \mathrm{C}$ NMR (100 MHz, $\left.\mathrm{CDCl}_{3}\right) \square$ 199.3, 154.1, 149.7, 148.7, 130.5, 126.3, 124.9, 122.8, 120.0, 118.4, 111.2, 37.4, 26.4, 22.5, 10.4; FTIR $\left(\mathrm{CHCl}_{3}\right) \mathrm{cm}^{-1}$ 3011, 1654, 1594, 1256; HRMS (EI) $m / z$ calcd for $\mathrm{C}_{15} \mathrm{H}_{14} \mathrm{O}_{2} 226.0994$, found 226.1004.

3-(2,4,5-Trimethoxyphenyl)-2-cyclopenten-1-one (Table 2, entry 1)

$\mathrm{R}_{f} 0.3$ (hexanes:EtOAc $\left.=1: 1\right) ; \mathrm{mp}=126{ }^{\circ} \mathrm{C} ;{ }^{1} \mathrm{H} \mathrm{NMR}\left(400 \mathrm{MHz}, \mathrm{CDCl}_{3}\right) \square 7.03(\mathrm{~s}$, $1 \mathrm{H}), 6.83(\mathrm{t}, 1 \mathrm{H}, J=1.6 \mathrm{~Hz}), 6.55(\mathrm{~s}, 1 \mathrm{H}), 3.95(\mathrm{~s}, 3 \mathrm{H}), 3.90(\mathrm{~s}, 3 \mathrm{H}), 3.87(\mathrm{~s}, 3 \mathrm{H})$, 3.06-3.03 (m, 2H), 2.48-2.46 (m, 2H); $\left.{ }^{13} \mathrm{C} \mathrm{NMR} \mathrm{(100} \mathrm{MHz,} \mathrm{CDCl}_{3}\right) \square$ 210.8, 169.7, 154.7, 152.4, 142.8, 129.6, 114.8, 112.1, 96.7, 56.7, 56.0, 55.9, 34.1, 30.4; FTIR $\left(\mathrm{CHCl}_{3}\right) \mathrm{cm}^{-1} 3011,1671,1563,1511,1466,1262,1216,1031$; HRMS (EI) $\mathrm{m} / \mathrm{z}$ calcd for $\mathrm{C}_{14} \mathrm{H}_{16} \mathrm{O}_{4} 248.1049$, found 248.1053.

3-(3-Methylbenzofuran-2-yl)-2-cyclopenten-1-one (Table 2, entry 2)

$\mathrm{R}_{f} 0.5$ (hexanes:EtOAc $\left.=2: 1\right) ; \mathrm{mp}=144{ }^{\circ} \mathrm{C} ;{ }^{1} \mathrm{H}$ NMR $\left(400 \mathrm{MHz}, \mathrm{CDCl}_{3}\right) \square 7.56(\mathrm{dd}$, $1 \mathrm{H}, J=0.7,7.3 \mathrm{~Hz}), 7.45(\mathrm{dd}, 1 \mathrm{H}, J=0.7,8.1 \mathrm{~Hz}), 7.38(\mathrm{ddd}, 1 \mathrm{H}, J=1.1,7.7,7.7 \mathrm{~Hz})$, $7.27(\mathrm{ddd}, 1 \mathrm{H}, J=1.1,7.5,7.5 \mathrm{~Hz}), 6.52(\mathrm{~d}, 1 \mathrm{H}, J=1.1 \mathrm{~Hz}), 3.13-3.10(\mathrm{~m}, 2 \mathrm{H})$, 2.54-2.51 (m, 2H), $2.45(\mathrm{~s}, 3 \mathrm{H}) ;{ }^{13} \mathrm{C}$ NMR (100 MHz, $\left.\mathrm{CDCl}_{3}\right) \square 208.6,162.9,154.5$, 147.0, 130.0, 127.4, 127.0, 122.9, 120.8, 120.2, 111.4, 34.3, 28.5, 9.7; FTIR $\left(\mathrm{CHCl}_{3}\right)$ $\mathrm{cm}^{-1}$ 3011, 1693, 1674, 1603, 1280, 1184; HRMS (TOF) $\mathrm{m} / \mathrm{z}$ calcd for $\mathrm{C}_{14} \mathrm{H}_{12} \mathrm{O}_{2}$ 213.0951, found 213.0925.

3-(2,4-Dimethoxyphenyl)-2-cyclohepten-1-one (Table 2, entry 3)

$\mathrm{R}_{f} 0.3$ (hexanes:EtOAc = 4:1); ${ }^{1} \mathrm{H}$ NMR $\left(400 \mathrm{MHz}, \mathrm{CDCl}_{3}\right) \square 7.09-7.06(\mathrm{~m}, 1 \mathrm{H})$, 6.45-6.43 (m, 2H), $6.09(\mathrm{~s}, 1 \mathrm{H}), 3.802(\mathrm{~s}, 3 \mathrm{H}), 3.798(\mathrm{~s}, 3 \mathrm{H}), 2.75-2.72(\mathrm{~m}, 2 \mathrm{H})$, 2.66-2.63 (m, 2H), 1.94-1.81 (m, 4H); $\left.{ }^{13} \mathrm{C} \mathrm{NMR} \mathrm{(100} \mathrm{MHz,} \mathrm{CDCl}_{3}\right) \square 204.5,161.2$, 159.0, 157.5, 131.5, 129.4, 125.7, 104.1, 98.8, 55.3, 55.3, 41.8, 32.5, 24.8, 20.8; FTIR (neat film) $\mathrm{cm}^{-1}$ 2941, 1652, 1606, 1504, 1463, 1456, 1300, 1261, 1209, 1160, 1031, 835; HRMS (EI) $m / z$ calcd for $\mathrm{C}_{15} \mathrm{H}_{18} \mathrm{O}_{3} 246.1256$, found 246.1254 . 
(士)-3-(2,4-Dimethoxyphenyl)-4-isopropyl-2-cyclohexen-1-one (Table 2, entry 4) $\mathrm{R}_{f} 0.6$ (hexanes:EtOAc $\left.=2: 1\right) ;{ }^{1} \mathrm{H}$ NMR $\left(400 \mathrm{MHz}, \mathrm{CDCl}_{3}\right) \square 7.04(\mathrm{~d}, 1 \mathrm{H}, J=8.4 \mathrm{~Hz})$, $6.46(\mathrm{dd}, 1 \mathrm{H}, J=2.2,8.4 \mathrm{~Hz}), 6.43(\mathrm{~d}, 1 \mathrm{H}, J=2.2 \mathrm{~Hz}), 6.02(\mathrm{~d}, 1 \mathrm{H}, J=1.8 \mathrm{~Hz}), 3.79(\mathrm{~s}$, $3 \mathrm{H}), 3.77(\mathrm{~s}, 3 \mathrm{H}), 3.03-2.98(\mathrm{~m}, 1 \mathrm{H}), 2.55-2.48(\mathrm{~m}, 1 \mathrm{H}), 2.43-2.35(\mathrm{~m}, 1 \mathrm{H}), 2.11-2.03$ $(\mathrm{m}, 1 \mathrm{H}), 1.95-1.79(\mathrm{~m}, 2 \mathrm{H}), 0.86(\mathrm{~d}, 3 \mathrm{H}, J=7.0 \mathrm{~Hz}), 0.66(\mathrm{~d}, 3 \mathrm{H}, J=7.0 \mathrm{~Hz}) ;{ }^{13} \mathrm{C}$ NMR (100 MHz, $\left.\mathrm{CDCl}_{3}\right) \square 200.1,165.6,161.2,157.1,129.7,129.3,122.3,104.5,98.5$, 55.3, 55.2, 43.3, 35.9, 29.6, 22.2, 21.3, 17.7; FTIR (neat film) $\mathrm{cm}^{-1} 2958,1668,1607$, $1504,1304,1250,1209,1160,1031,835$; HRMS (TOF) $\mathrm{m} / z$ calcd for $\mathrm{C}_{17} \mathrm{H}_{22} \mathrm{O}_{3}$ 275.1647 , found 275.1652 .

(士)-3-(3-Methylbenzofuran-2-yl)-4-isopropyl-2-cyclohexen-1-one (Table 2, entry 5) $\mathrm{R}_{f} 0.6$ (hexanes:EtOAc $\left.=3: 1\right) ; \mathrm{mp}=124{ }^{\circ} \mathrm{C} ;{ }^{1} \mathrm{H}$ NMR $\left(400 \mathrm{MHz}, \mathrm{CDCl}_{3}\right) \square 7.56(\mathrm{dd}$, $1 \mathrm{H}, J=0.7,8.4 \mathrm{~Hz}), 7.46(\mathrm{~d}, 1 \mathrm{H}, J=8.4 \mathrm{~Hz}), 7.35(\mathrm{ddd}, 1 \mathrm{H}, J=1.1,7.1,7.1 \mathrm{~Hz}), 7.27$ $(\mathrm{dd}, 1 \mathrm{H}, J=7.5,7.5 \mathrm{~Hz}), 6.39(\mathrm{~s}, 1 \mathrm{H}), 3.11-3.07(\mathrm{~m}, 1 \mathrm{H}), 2.67-2.58(\mathrm{~m}, 1 \mathrm{H}), 2.47-2.41$ $(\mathrm{m}, 1 \mathrm{H}), 2.43(\mathrm{~s}, 3 \mathrm{H}), 2.28-2.11(\mathrm{~m}, 2 \mathrm{H}), 2.03-1.94(\mathrm{~m}, 1 \mathrm{H}), 0.99(\mathrm{~d}, 3 \mathrm{H}, J=7.0 \mathrm{~Hz})$, $0.87(\mathrm{~d}, 3 \mathrm{H}, J=7.0 \mathrm{~Hz}) ;{ }^{13} \mathrm{C}$ NMR $\left(100 \mathrm{MHz}, \mathrm{CDCl}_{3}\right) \square 199.5,154.2,153.9,149.8$, 130.4, 126.4, 125.9, 122.8, 120.0, 117.2, 111.2, 40.4, 33.9, 31.1, 23.8, 21.2, 20.6, 10.3; FTIR (neat film) $\mathrm{cm}^{-1} 2960,1666,1589,1453,1331,1252,1102,879,747$; HRMS (TOF) $\mathrm{m} / \mathrm{z}$ calcd for $\mathrm{C}_{18} \mathrm{H}_{20} \mathrm{O}_{2} 269.1541$, found 269.1539 .

3-(2,4-Dimethoxyphenyl)-4,4-dimethyl-2-cyclohexen-1-one (Table 2, entry 6) $\mathrm{R}_{f} 0.5$ (hexanes:EtOAc $\left.=2: 1\right) ;{ }^{1} \mathrm{H} \mathrm{NMR}\left(400 \mathrm{MHz}, \mathrm{CDCl}_{3}\right) \square 6.85(\mathrm{~d}, 1 \mathrm{H}, J=7.7 \mathrm{~Hz})$, 6.46-6.43 (m, 3H), $5.73(\mathrm{~s}, 1 \mathrm{H}), 3.80(\mathrm{~s}, 3 \mathrm{H}), 3.72(\mathrm{~s}, 3 \mathrm{H}), 2.55(\mathrm{t}, 2 \mathrm{H}, J=6.8 \mathrm{~Hz}), 1.97$ $(\mathrm{t}, 2 \mathrm{H}, J=6.8 \mathrm{~Hz}), 1.10(\mathrm{~s}, 6 \mathrm{H}) ;{ }^{13} \mathrm{C}$ NMR $\left(100 \mathrm{MHz}, \mathrm{CDCl}_{3}\right) \square$ 199.6, 168.6, 160.5, 157.0, 129.3, 129.0, 121.3, 103.5, 98.5, 55.3, 55.2, 38.2, 36.4, 34.5, 26.7; FTIR (neat film) $\mathrm{cm}^{-1} 2963,1673,1614,1579,1505,1463,1304,1264,1209,1159,1131,1032$, 834; HRMS (TOF) $m / z$ calcd for $\mathrm{C}_{16} \mathrm{H}_{20} \mathrm{O}_{3}$ 261.1490, found 261.1487.

\section{3-(2,4-Dimethoxyphenyl)-2-(2,4-dimethoxybenzyl)-2-cyclopenten-1-one (2)}

$\mathrm{R}_{f} 0.5$ (hexanes:EtOAc $\left.=1: 1\right) ;{ }^{1} \mathrm{H} \mathrm{NMR}\left(400 \mathrm{MHz}, \mathrm{CDCl}_{3}\right) \square 6.99(\mathrm{~d}, 1 \mathrm{H}, J=8.4 \mathrm{~Hz})$, $6.88(\mathrm{~d}, 1 \mathrm{H}, J=8.8 \mathrm{~Hz}), 6.46(\mathrm{~d}, 1 \mathrm{H}, J=2.2 \mathrm{~Hz}), 6.43(\mathrm{dd}, 1 \mathrm{H}, J=2.2,8.4 \mathrm{~Hz})$, 6.33-6.31 (m, 2H), $3.80(\mathrm{~s}, 3 \mathrm{H}), 3.73(\mathrm{~s}, 3 \mathrm{H}), 3.69(\mathrm{~s}, 3 \mathrm{H}), 3.62(\mathrm{~s}, 3 \mathrm{H}), 3.43(\mathrm{~s}, 2 \mathrm{H})$, 2.95-2.92 (m, 2H), 2.53-2.51 (m, 2H); $\left.{ }^{13} \mathrm{C} \mathrm{NMR} \mathrm{(100} \mathrm{MHz,} \mathrm{CDCl}_{3}\right) \square$ 209.6, 169.4, $161.3,158.9,157.8,157.5,139.8,129.3,129.1,119.8,118.8,104.1,103.4,98.5,97.9$, 55.3, 55.2, 55.1, 54.9, 34.4, 30.7, 23.3; FTIR (neat film) $\mathrm{cm}^{-1}$ 2937, 1694, 1608, 1505, $1294,1209,1158,1035,829$; HRMS (TOF) $\mathrm{m} / z$ calcd for $\mathrm{C}_{22} \mathrm{H}_{24} \mathrm{O}_{5} 369.1702$, found 369.1716 .

(E)-3-(2,4-Dimethoxyphenyl)-2-(2,4-dimethoxybenzylidene)-cyclopentan-1-one (3) $\mathrm{R}_{f} 0.6$ (hexanes:EtOAc $\left.=1: 1\right) ;{ }^{1} \mathrm{H}$ NMR $\left(400 \mathrm{MHz}, \mathrm{CDCl}_{3}\right) \square 8.03(\mathrm{~s}, 1 \mathrm{H}), 7.01(\mathrm{~d}, 1 \mathrm{H}$, $J=8.4 \mathrm{~Hz}), 6.82(\mathrm{~d}, 1 \mathrm{H}, J=8.1 \mathrm{~Hz}), 6.53(\mathrm{~d}, 1 \mathrm{H}, J=1.8), 6.38(\mathrm{~d}, 1 \mathrm{H}, J=2.2 \mathrm{~Hz})$, $6.30(\mathrm{dd}, 1 \mathrm{H}, J=2.2,8.4 \mathrm{~Hz}), 6.25(\mathrm{dd}, 1 \mathrm{H}, J=1.8,8.8 \mathrm{~Hz}), 4.59(\mathrm{~d}, 1 \mathrm{H}, J=7.7 \mathrm{~Hz})$, $3.90(\mathrm{~s}, 3 \mathrm{H}), 3.83(\mathrm{~s}, 3 \mathrm{H}), 3.77(\mathrm{~s}, 3 \mathrm{H}), 3.76(\mathrm{~s}, 3 \mathrm{H}), 2.42-2.18(\mathrm{~m}, 3 \mathrm{H}), 2.05-1.97(\mathrm{~m}$, 
$1 \mathrm{H}) ;{ }^{13} \mathrm{C}$ NMR $\left(100 \mathrm{MHz}, \mathrm{CDCl}_{3}\right) \square 208.9,162.2,160.6,159.6,157.7,135.8,131.4$, $128.3,127.7,124.0,116.9,104.7,103.5,99.1,98.0,55.5,55.4,55.3,55.3,40.0,35.4$, 29.0; FTIR (neat film) $\mathrm{cm}^{-1} 2940,1704,1597,1503,1463,1293,1260,1210,1160$, $1116,1033,833$; HRMS (TOF) $m / z$ calcd for $\mathrm{C}_{22} \mathrm{H}_{24} \mathrm{O}_{5} 369.1702$, found 369.1698 . 